\title{
Erros de medicação: tipos, fatores causais e providências tomadas em quatro hospitais brasileiros
}

\author{
MEDICATION ERRORS: TYPES, CAUSES AND MEASURES TAKEN IN FOUR BRAZILIAN HOSPITALS \\ ERRORES EN LA MEDICACIÓN: TIPOS, FACTORES CAUSALES Y PROVIDENCIAS TOMADAS \\ EN CUATRO HOSPITALES BRASILEÑOS
}

\section{Adriana Inocenti Miasso', Cris Renata Grou², Silvia Helena De Bortoli Cassiani, Ana Elisa Bauer de Camargo Silva ${ }^{4}$, Flávio Trevisan Fakih ${ }^{5}$}

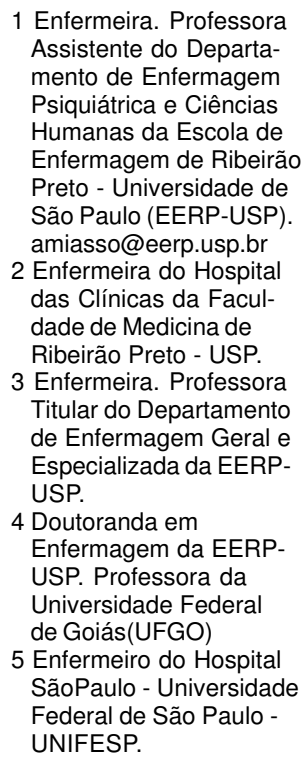

3 Enfermeira. Professora Titular do Departamento de Enfermagem Geral e Especializada da EERP. USP.

4 Doutoranda em

Enfermagem da EERPUSP. Professora da Universidade Federal de Goiás(UFGO)

5 Enfermeiro do Hospital SãoPaulo - Universidade Federal de São Paulo UNIFESP.

\section{RESUMO}

Este estudo analisou, em quatro hospitais brasileiros, tipos, causas, providências administrativas tomadas e sugestões, em relação aos erros na medicação, na perspectiva dos profissionais envolvidos no sistema de medicação. Trata-se de um estudo multicêntrico, do tipo survey exploratório. A amostra constou de profissionais de clínica médica e farmácia, dos referidos hospitais. Para coleta de dados, utilizou-se a técnica de entrevista semi-estruturada. Os resultados evidenciaram que os tipos de erros mais citados pelos profissionais foram aqueles relacionados à prescrição/transcrição dos medicamentos. A falta de atenção, falhas individuais e problemas na administração dos serviços constituíram importantes atributos das causas dos erros. Relatórios foram as principais providências tomadas ante os erros e mudanças nas atitudes individuais as mais citadas como forma de prevení-los.

\section{ABSTRACT}

This study analyzed the causes, types, administrative measures taken and suggestions concerning medication errors according to the perspective of professionals involved with the medication systems in four Brazilian hospitals. It is an exploratory, survey-type, multicentric study. The sample consisted of professionals from the medical clinic and pharmacy in the abovementioned hospitals. Semistructured interviews were used for data collection. Results showed that the most frequently error types mentioned by the professionals were related to medication ordering/transcription. Lack of attention, individual mistakes and problems in service management were the major causes of errors. Reports were the main measures taken in view of errors, and changes in individual attitudes were the most frequently mentioned form to prevent them.

\section{KEY WORDS}

Medication errors.

Hospital administration.

Medication systems.

\section{RESUMEN}

E este estudio se analizó, en cuatro hospitales brasileños, tipos, causas, providencias administrativas tomadas y sugerencias, en relación a errores en la medicación, en la perspectiva de los profesionales involucrados en el sistema de medicación. Se trata de un estudio multicéntrico, del tipo survey exploratorio. La muestra estuvo constituida por profesionales de la clínica médica y farmacia, de los referidos hospitales. Para la recolección de datos, se utilizó la técnica de la entrevista semiestructurada. Los resultados evidenciaron que los tipos de errores más citados por los profesionales fueron aquellos relacionados a la prescripción/ transcripción de los medicamentos. La falta de atención, fallas individuales y problemas en la administración de los servicios constituyeron importantes atributos de las causas de los errores. Los informes fueron las principales providencias tomadas ante los errores y los cambios en las actitudes individuales las más citadas como forma de prevenirlos.

\section{DESCRIPTORES}

Errores de medicación. Administración hospitalaria. Sistemas de medicación. 


\section{INTRODUÇÃO}

Os erros de medicação representam uma triste realidade no trabalho dos profissionais de saúde, com sérias conseqüências para pacientes e organização hospitalar ${ }^{(1)}$.

Estudo desenvolvido nos EUA, em 36 hospitais e instalações de saúde, identificou como erros mais freqüentes na administração de medicamentos: horário errado (43\%), omissão (30\%), dose errada (17\%) e aplicação de medicamento sem autorização $(4 \%)^{(2)}$.

Cerca de $30 \%$ dos danos durante a hospitalização estão associados a erros na medicação, os quais trazem, também, sérias conseqüências econômicas às instituições de saúde. $\mathrm{O}$ custo anual de morbidade e mortalidade referente a erros na medicação, nos EUA, tem sido estimado em torno de US\$ 76,6 bilhões ${ }^{(3-5)}$.

A abordagem comum nas instituições e os esforços tradicionais na redução dos erros na medicação enfocam os indivíduos, mais freqüentemente, os profissionais da enfermagem por realizarem a administração, propriamente dita, do medicamento. Não são raras as práticas da punição, suspensão e demissão, ou seja, a culpa recai no indivíduo que cometeu ou que não percebeu um erro já iniciado ${ }^{(6-7)}$.

Medicar pacientes depende de ações meramente humanas e os erros fazem parte dessa natureza, porém, um sistema de medicação bem estruturado deverá promover condições que auxiliem na minimização e prevenção dos erros, implementando normas, regras, ações, processos com a finalidade de auxiliar os profissionais envolvidos.

Assim, especialistas e investigadores que há muito vêm se dedicando ao estudo dessa temática - erros na medicação - são unânimes, e não há mais dúvidas, em rejeitar as abordagens que enfocam os indivíduos puramente como causa de erros e notificam que mudanças no sistema de medicação constituem estratégias efetivas para reduzir a probabilidade de acidentes ${ }^{(8-9)}$.

Sabe-se que são várias as estratégias a serem implantadas no sistema de medicação desenvolvido pela administração de um hospital, com o intuito de levar o medicamento até o paciente de maneira segura. Acreditamos que a identificação dos tipos de erros mais freqüentes bem como, dos fatores causais podem contribuir para que os mesmos sejam revertidos em educação e melhorias para o sistema.

\section{OBJETIVO}

Identificar e analisar, em quatro hospitais de diferentes regiões brasileiras, os tipos, causas, providências administrativas e sugestões a respeito de erros na medicação, na perspectiva dos profissionais envolvidos no sistema de medicação.

\section{METODOLOGIA}

Trata-se de um estudo multicêntrico, do tipo survey exploratório, que utiliza a abordagem quali-quantitativa. Foi realizado em unidades de clínica médica e farmácia hospitalar de 04 hospitais universitários localizados nas cidades de Recife, Ribeirão Preto, Goiânia e São Paulo, tendo como foco a problemática dos erros no processo de administração de medicamentos desenvolvido nestes hospitais, descritos no texto como Hospitais A, B, C e D e não na correspondência com as cidades citadas.

Estas instituições foram escolhidas por possuírem vínculo com universidades públicas estaduais ou federais, por fazerem parte da Rede Sentinela da ANVISA e por serem campo de estágio para as instituições formadoras de profissionais de saúde do país. A unidade de clínica médica foi selecionada por possuir, em média, leitos reservados a pacientes portadores de doenças crônico-degenerativas que, usualmente, utilizam-se de grande e variada gama de medicamentos em seu tratamento, por longo período.

A população constou de todos os profissionais que pertenciam ao quadro de pessoal da clínica médica e da farmácia hospitalar, envolvidos com o sistema de medicação, nos quatro hospitais em estudo. A amostra foi constituída por 152 profissionais que atuavam na clínica médica e farmácia hospitalar, nos quatro hospitais em estudo, no período da investigação e que consentiram, por escrito, serem entrevistados.

Após aprovação do estudo no Comitê de Ética em Pesquisa dos quatro hospitais investigados e consentimento escrito dos profissionais, deu-se início a coleta dos dados. Para obtenção dos mesmos foram realizadas entrevistas semiestruturadas, previamente agendadas com os profissionais envolvidos no sistema de medicação, nos meses de setembro a dezembro de 2002, com intuito de obter uma visão geral do sistema vigente. Para tanto, foi utilizado um roteiro contendo dados referentes a: categoria profissional e função dos entrevistados, tipos de erros ou potencial para erros na medicação mais freqüentes, motivo da ocorrência dos erros, providências tomadas mediante a mesma e sugestões para prevenir erros na medicação. Os dados foram registrados no roteiro pelas próprias pesquisadoras. A seguir foram digitados e, após lê-los, os categorizamos por semelhança de conteúdo e os analisamos, sendo os mesmos apresentados em tabelas, contendo freqüências absolutas e relativas, e em forma de depoimentos expressos pelos entrevistados.

\section{RESULTADOS E DISCUSSÃO}

Um total de 152 pessoas foi entrevistado. Dessas, 25 eram do hospital A, 24 do hospital B, 40 do hospital C e 62 do hospital D. Os resultados obtidos são apresentados a seguir, distribuídos por categorias de profissionais, utilizando para este fim siglas como: médicos (M), enfermeiros (E) etc, conforme Quadro 1. 
Quadro 1 - Distribuição dos profissionais entrevistados por hospital e categoria profissional (Goiânia, Recife, Ribeirão Preto, São Paulo) - 2002

\begin{tabular}{|c|c|c|c|c|c|}
\hline $\begin{array}{ll}\text { CATEGORIAS } \\
\text { PROFISSIONAIS }\end{array}$ & $\begin{array}{l}\text { Sigla usada } \\
\text { no estudo }\end{array}$ & HOSP A & HOSP B & HOSP C & HOSP D \\
\hline $\begin{array}{l}\text { Médicos } \\
\text { Enfermeiros } \\
\text { Técnicos de enfermagem } \\
\text { Auxiliares de enfermagem } \\
\text { Auxiliar administrativo } \\
\text { Escriturário } \\
\text { Farmacêutico } \\
\text { Residente de farmácia } \\
\text { Atendente de farmácia } \\
\text { Auxiliar de farmácia } \\
\text { Técnico de farmácia }\end{array}$ & $\begin{array}{c}\text { M } \\
\text { E } \\
\text { TE } \\
\text { AE } \\
\text { AA } \\
\text { ES } \\
\text { F } \\
\text { RF } \\
\text { ATF } \\
\text { AF } \\
\text { TF }\end{array}$ & $\begin{array}{r}5 \\
5 \\
1 \\
12 \\
1 \\
2 \\
- \\
- \\
- \\
- \\
-\end{array}$ & $\begin{array}{l}4 \\
7 \\
1 \\
7 \\
- \\
- \\
1 \\
- \\
- \\
4 \\
-\end{array}$ & $\begin{array}{r}12 \\
4 \\
14 \\
2 \\
- \\
- \\
3 \\
- \\
- \\
- \\
5\end{array}$ & $\begin{array}{c}13 \\
6 \\
- \\
26 \\
- \\
- \\
6 \\
1 \\
3 \\
7 \\
-\end{array}$ \\
\hline
\end{tabular}

Vale ressaltar que, a partir desse momento, estas siglas (categorias profissionais) serão utilizadas na apresentação dos resultados do estudo. Antecede a elas o número referente ao profissional entrevistado (de acordo com a seqüência das entrevistas) e, segue-se as mesmas, as letras referentes aos hospitais investigados (A,B,C e D).
A Tabela 1 apresenta a distribuição das categorias dos erros ou potencial para erros relacionados à medicação, nos quatro hospitais investigados, segundo a opinião dos entrevistados.

Tabela 1 - Distribuição das categorias dos erros ou potencial para erros relacionados à medicação nos quatros hospitais investigados, segundo a opinião dos entrevistados (Goiânia, Recife, Ribeirão Preto, São Paulo) - 2002

\begin{tabular}{|c|c|c|c|c|c|c|c|c|c|c|}
\hline \multirow{2}{*}{ CATEGORIA } & \multicolumn{2}{|c|}{ Hospital A } & \multicolumn{2}{|c|}{ Hospital B } & \multicolumn{2}{|c|}{ Hospital C } & \multicolumn{2}{|c|}{ Hospital D } & \multicolumn{2}{|c|}{ Hospital E } \\
\hline & $\mathbf{n}$ & $\%$ & $\mathrm{n}$ & $\%$ & $\mathrm{n}$ & $\%$ & $\mathrm{n}$ & $\%$ & $\mathrm{n}$ & $\%$ \\
\hline Erros relacionados ao paciente & 2 & 3,3 & 1 & 1,3 & 7 & 4,5 & 6 & 4,7 & 16 & 3,8 \\
\hline Erros relacionados ao horário & 12 & 19,7 & 5 & 6,3 & 32 & 20,6 & 8 & 6,2 & 57 & 13,5 \\
\hline Erros relacionados à prescrição/transcrição & 14 & 23 & 25 & 31,6 & 45 & 29,0 & 42 & 32,8 & 126 & 29,5 \\
\hline Erros relacionados à solicitação & 5 & 8,2 & 2 & 2,5 & 8 & 5,2 & 4 & 3,1 & 19 & 4,5 \\
\hline Erros relacionados a dispensação do medicamento & 3 & 4,9 & 24 & 30,4 & 20 & 12,9 & 25 & 19,5 & 72 & 17,0 \\
\hline $\begin{array}{l}\text { Erros relacionados ao preparo e administração de } \\
\text { medicamentos }\end{array}$ & 6 & 9,8 & 11 & 13,9 & 21 & 13,5 & 32 & 25 & 70 & 16,5 \\
\hline Erros relacionados ao registro do medicamento & 1 & 1,6 & 0 & 0 & 18 & 11,6 & 4 & 3,1 & 23 & 5,4 \\
\hline Erros por falhas de comunicação & 12 & 19,7 & 2 & 2,5 & 3 & 1,9 & 2 & 1,6 & 19 & 4,5 \\
\hline Erros desencadeados por desorganização da unidade & 6 & 9,84 & - & - & - & - & - & - & 6 & 1,42 \\
\hline Outros & - & - & 9 & 11,4 & 1 & 0,6 & 5 & 3,9 & 15 & 3,5 \\
\hline Total & 61 & 100 & 79 & 100 & 155 & 100 & 128 & 100 & 423 & 100 \\
\hline
\end{tabular}

As respostas obtidas evidenciam que os tipos de erros mais citados pelos profissionais foram àqueles relacionados à prescrição/transcrição de medicamentos, em todos hospitais investigados. A esse respeito, evidencia-se que os erros devem-se à dificuldade de compreensão da letra do médico (no caso de prescrições manuais) e a erros ou inadequação da prescrição, conforme pode ser evidenciado nos relatos a seguir.

Assim, primeiro eles acham que a gente é adivinho, porque as letras são uns garranchos e as medicações, principalmente antibióticos, tem uns nomes muito idênticos (cefazolina e cefalotina), os que você vê mal escrito, você confunde mesmo. Aí é hora de ir medicação errada. (1TFC)

Medicamentos que não precisam ser administrados e que o são (ex: ranitidina para todos os pacientes). (1MB)

Medicamentos para HAS e o paciente encontra-se hipotenso. (3EB)

Prescrição médica rasurada. (2EB)

Os transtornos acarretados nas atividades da farmácia e da enfermagem, com as idas e vindas de pessoas, devido à caligra- 
fia ruim do médico, ocasionam atrasos e acabam gerando gastos desnecessários às instituições. Esta situação agrava-se quando, na ausência do médico responsável pela prescrição para esclarecer dúvidas acerca da mesma, o profissional interpreta a prescrição de acordo com sua experiência e dispensa ou administra o medicamento que acredita estar prescrito.

A prescrição médica eletrônica constitui uma das estratégias citadas na literatura ${ }^{(10)}$ para prevenir ou reduzir erros na medicação, uma vez que resolve a dificuldade relativa às letras ilegíveis. Entretanto, é um sistema caro, mesmo para os padrões internacionais e, assim, nem todas as instituições podem contar com um sistema implementado ${ }^{(11)}$. Nesse caso, algumas atividades devem ser padronizadas nas instituições como evitar abreviações, não usar decimais, utilização de letras claras e legíveis, não misturar nas prescrições nomes genéricos e comerciais de medicamentos, entre outras.

Prescrições incorretas, feitas principalmente por alunos da medicina, são um fato. A enfermagem tem, nesse caso, um aspecto importante atuando, muitas vezes, como barreira para erros. Os depoimentos a seguir exemplificam o fato.

Via errada também ocorre. Aconteceu de ficarem 3 dias prescrevendo cabeceira elevada SC. Quer dizer, a pessoa nem lia o que estava escrevendo. Então nem presta atenção no que está fazendo, nem lê. É uma cópia, e às vezes até uma cópia errada, porque sei lá uma insulina SC, cabeceira elevada, emenda as duas faz uma só e nem pensa no que está escrevendo. Então a gente já pegou muitas vezes. (1EC)

Quando é agora, em fevereiro, começa turma nova de residentes. Aí acontece muito erro. Erro de prescrição do tipo Fungizon diluído em SF 0,9\% quando só pode ser diluído em Soro Glicosado. Dosagem mais alta do que o recomendado. Prescrevem dolantina 'Ah, faz uma ampola de dolantina', dolantina é outro também que você não faz, você dilui ela pra 10 e faz 2 ou $3 \mathrm{ml}$, não mais do que isso. (2AEC)

As prescrições de medicamentos são o início de uma cadeia de ações que levará o medicamento até o paciente, não podendo constituir um fator exacerbado para o erro ou potencial para erro na medicação. Este constitui um aspecto preocupante uma vez que os depoimentos, acima mencionados, refletem, ainda, a carência de conhecimento do médico acerca dos medicamentos prescritos. A esse respeito, estudo ${ }^{(12)}$ evidencia que os médicos, devido à formação acadêmica inadequada em farmacologia, geralmente não possuem as noções básicas necessárias para melhor atuação profissional e que sem tais noções, não é possível manipular adequadamente a farmacoterapia, aumentando a probabilidade da ocorrência de iatrogenias.

Aliado a esse contexto, tem-se que, infelizmente, nem sempre a enfermagem consegue atuar enquanto barreira para os erros de medicação e, por atuar na última etapa do sistema de medicação, que consiste na administração propriamente dita do medicamento, geralmente assume o ônus de muitos erros cometidos no início ou no meio do sistema e não detectados.
Com relação ao horário, a percepção dos entrevistados denota a preocupação com os atrasos do medicamento para chegar ao andar, demorando-se para iniciar antibióticos e anti-hipertensivos, principalmente. Tais atrasos ocorrem tanto devido à farmácia quanto ao próprio trabalho da enfermagem, acarretando administração da medicação em horário errado, conforme evidenciam os relatos a seguir.

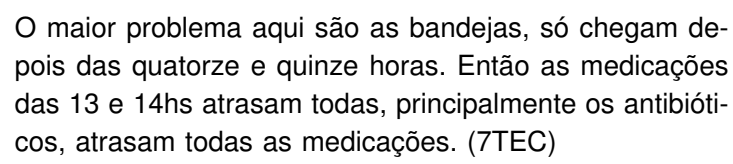

Ocorre geralmente de algumas prescrições estarem assim: fazer Lasix as 10 e às 16 horas, mas às $10 \mathrm{~h}$ a medicação ainda não chegou. Vai chegar á tarde. Então as das 10 horas fica sem fazer, e vai para o dia seguinte. (1EC)

A importância de se seguir rigorosamente os intervalos de tempo entre as doses administradas está relacionada aos eventos farmacocinéticos e à farmacodinâmica que fundamentam a necessidade da dosagem seguir uma certa sequiência no tempo, para que a ação do medicamento seja mantida ${ }^{(13)}$.

Erros devido à falta do medicamento ou falhas na comunicação são muito citados pelos entrevistados. O principal deles é a suspensão do medicamento pelo médico que não avisa a enfermagem. Os depoimentos a seguir mostram que as queixas vêm tanto da equipe de enfermagem como da equipe médica.

Quando a freqüência da medicação é alterada pelo médico, por exemplo, de $6 \times 6 \mathrm{~h}$ para $8 \times 8 \mathrm{~h}$, e o mesmo não avisa. (3AAA)

A gente prescreve os antibióticos, por exemplo, porque a gente acredita que tenha e tem época que o hospital tem deficiência desses medicamentos. A auxiliar de enfermagem que está com o paciente, às vezes não olha, ela que é obrigada a olhar, pegar o kit para ver se tem ou não, e ela não avisa, não avisou quando não tinha, e o paciente ficou sem a medicação. Isso aí foi um erro muito grave. Quando a medicação é de urgência, por exemplo, antihipertensivo, que eu acho uma medicação muito importante, às vezes passa batido. (7MC)

Estudo de referência que analisou o sistema de medicação de uma instituição hospitalar, identificou 16 falhas no mesmo, sendo a dificuldade na comunicação entre pessoal de diferentes serviços uma delas ${ }^{(14)}$. Sabe-se que um processo de comunicação eficaz é indispensável para a garantia da segurança do paciente na administração de seus medicamentos. Falhas nesse processo podem resultar em piora na condição do paciente, atraso de sua recuperação e, até mesmo, em óbito.

No hospital B é possível identificar que se segue aos erros relacionados à prescrição/transcrição, os relacionados a dispensação dos medicamentos. A farmácia não envia o medicamento por falta, havendo, também, falhas na identificação do mesmo, quantidade insuficiente, medicamentos trocados, etc, conforme mostram os depoimentos a seguir. 
Falta muita medicação, falta demais!! Às vezes a farmácia teima que mandou, a gente precisa guardar o Kit lacrado pra mostrar pra eles que não veio. Mas aconteceu isso demais, principalmente antibiótico. (6TEC)

Comprimidos sem identificação (blísters cortados, sem nome, sem data, sem dose. (1AFB)

Troca: furosemida e ranitidina frascos iguais sendo enviados trocados. (1FB)

A farmacêutica não aceita que tem erros. Mas assim, é ranitidina VO e vem EV e tem muito!! Esses dias veio Cipro no lugar de Maxcef, não tinha nada haver uma coisa com a outra, mas ela acha que não, a farmácia não tem erro. (2AEC)

As medicações devolvidas às vezes vêm com identificação feita de esparadrapo colado no rótulo do frasco, o que leva muitas vezes a danos no rótulo quando o esparadrapo é retirado. As medicações vêm misturadas (ampolas) o que dificulta a sua separação. (AFD)

Os erros mais comuns na dispensação de medicamentos envolvem doses ou formas incorretas, que podem ocorrer devido a distrações ou problemas com o ambiente de trabalho ${ }^{(15)}$, problemas que foram também encontrados nos hospitais em estudo.

Pode-se constatar, ainda, através da Tabela 1, que dos quatro hospitais investigados, o hospital A foi o que apresentou menor percentual $(4,9 \%)$ de erros relacionados à dispensação de medicamentos. Vale ressaltar que apenas neste hospital funciona um sistema informatizado de medicação. Nesse sistema, cada medicamento (dose individualizada) deve conter um código de barras que ao ser passado por um visor óptico, na farmácia, caso não coincida com a prescrição feita no dia para o paciente, ocorre a emissão de um aviso na tela do computador de que o medicamento não consta na prescrição. Se houver, por exemplo, a prescrição de 10 comprimidos de um mesmo tipo prescrito para o paciente no dia, o código de barras de cada comprimido, individualmente, deve ser passado pelo visor óptico. Ocorre que, em algumas situações, o profissional responsável pela dispensação, neste hospital, passa o mesmo comprimido dez vezes pelo visor e, ao contar os comprimidos para dispensá-los acaba cometendo erros, o que justifica o percentual de erros mencionado acima.

Há relatos de erros relacionados ao preparo e à administração de medicamentos como: erros na diluição e dosagem, troca de vias, de medicamentos entre pacientes, problemas com a checagem, etc.

Medicamentos em cápsulas que erroneamente são abertos para passar por sonda. EX: Omeprazol.(1MA)

Às vezes a pessoa chega pra trabalhar à tarde e a medicação do período da manhã está sem checar. Aí você não sabe se foi feito ou não, fica difícil! (1TEC)

... a dosagem vem da farmácia diferente da prescrição e a gente tem que adequá-la. Às vezes está escrito $25 \mathrm{mg}$ e o comprimido vem de $200 \mathrm{mg}$. Tem que ter muita atenção tanto no preparo quanto na administração. (5TEC)

Os relatos acima denotam carência de conhecimento e/ou compromisso em relação à tarefa de administrar medicamentos. É importante que o profissional que participa da medicação de um paciente conheça seu papel na corrente que se forma no desempenho das tarefas, para que desenvolva tal papel com segurança, consciência, responsabilidade e eficiência. A esse respeito, estudo(1) desenvolvido propôs três estratégias para minimizar erros no preparo e administração do medicamento, quais sejam: cumprimento de políticas e procedimentos referentes ao preparo e à administração; educação dos profissionais e melhora na comunicação

Quando questionados sobre as causas dos erros relacionados à medicação, os entrevistados emitiram as opiniões que, organizadas, estão apresentadas na Tabela 2.

Tabela 2 - Distribuição das categorias de causas de erros relacionados a medicações em quatro hospitais, segundo a opinião dos entrevistados (Goiânia, Recife, Ribeirão Preto, São Paulo) - 2002

\begin{tabular}{|c|c|c|c|c|c|c|c|c|c|c|}
\hline \multirow{2}{*}{ CATEGORIA } & \multicolumn{2}{|c|}{ Hospital A } & \multicolumn{2}{|c|}{ Hospital B } & \multicolumn{2}{|c|}{ Hospital C } & \multicolumn{2}{|c|}{ Hospital D } & \multicolumn{2}{|c|}{ Total } \\
\hline & $\mathrm{n}$ & $\%$ & $\mathrm{n}$ & $\%$ & $\mathrm{n}$ & $\%$ & $\mathrm{n}$ & $\%$ & $\mathrm{n}$ & $\%$ \\
\hline Prescrições médicas & 3 & 8,6 & 0 & 0 & 5 & 6,6 & 12 & 11,5 & 20 & 7,5 \\
\hline Falhas individuais & 3 & 8,6 & 6 & 11,5 & 15 & 19,7 & 29 & 27,9 & 53 & 19,8 \\
\hline Falta de atenção & 16 & 45,7 & 13 & 25,0 & 21 & 27,6 & 12 & 11,5 & 62 & 23,2 \\
\hline Excesso de trabalho & 6 & 17,1 & 10 & 19,2 & 11 & 14,5 & 8 & 7,7 & 35 & 13,1 \\
\hline Gestão de pessoal & 1 & 2,9 & - & - & - & - & 9 & 8,6 & 10 & 3,7 \\
\hline $\begin{array}{l}\text { Falha na comunicação e integração de } \\
\text { pessoas e setores }\end{array}$ & 3 & 8,6 & 3 & 5,8 & 5 & 6,6 & 9 & 8,6 & 20 & 7,5 \\
\hline $\begin{array}{l}\text { Diversidade de medicamentos existentes } \\
\text { e suas identificações }\end{array}$ & - & - & 4 & 7,7 & - & - & 4 & 3,8 & 8 & 3,0 \\
\hline $\begin{array}{l}\text { Falhas na administração e organização } \\
\text { dos serviços }\end{array}$ & 3 & 8,5 & 15 & 28,9 & 10 & 13,2 & 17 & 16,3 & 45 & 16,8 \\
\hline Outros problemas & 0 & 0 & 1 & 1,9 & 9 & 11,8 & 4 & 3,8 & 14 & 5,2 \\
\hline Total & 35 & 100 & 52 & 100 & 76 & 100 & 104 & 100 & 267 & 100 \\
\hline
\end{tabular}


Nota-se que a falta de atenção é um atributo das causas dos erros, perpetuando a idéia de que o indivíduo, e não o sistema, é o responsável. Essa cultura determina, também, punições para os profissionais como relatórios, advertências, etc.

No que refere às falhas individuais como falta de conhecimento, pressa, falta de atenção, falta de treinamento, resistência do profissional médico em seguir protocolos são enumerados os seguintes depoimentos.

\begin{abstract}
A falta de interesse é tremenda, a pessoa tem que ter mais interesse, ela está trabalhando com pessoas que já estão doentes e vieram para o hospital tratar e não é justo que ela pegue uma infecção ainda. (1TFC)
\end{abstract}

O básico do pessoal da enfermagem é que eles vão ficando tão práticos que confiam demais no que fazem, e aí é onde eu acho que acontecem os erros! (2EC)

Falta de atenção da equipe de enfermagem na hora de colocar os horários; o médico altera a prescrição e a enfermeira não vê, portanto continua mantendo os mesmos horários anteriores. (3MB)

Solicitação constante de outras pessoas durante o processo de organização e preparo do medicamento. (4EB)

Muitos estudos têm responsabilizado os médicos prescritores pela maioria dos erros na medicação, e dois deles indicaram que os enganos ou lapsos foram as causas mais frequientes de erros $(57 \%)^{(16-17)}$. O que chama a atenção é o fato de os profissionais culparem a si mesmos pelos erros, deixando de lado a visão de que o sistema em que estão envolvidos pode, também, colaborar para sua ocorrência.

O excesso de trabalho, a falta de pessoal, o volume de tarefas, a carga horária pesada e o número de pacientes com grande número de medicações devem ser levados em conta. Também as falhas na comunicação e na integração dos setores entre os profissionais são citadas como determinantes na ocorrência dos erros. O depoimento a seguir, explicita uma situação.

Falta de comunicação entre os departamentos, entre os chefes das seções. Porque o médico chega de manhã nervoso, de um plantão agitado, pra prescrever, prescreve aquela beleza, trata a enfermeira como se ela fosse um animal. A enfermeira já liga pra farmácia brigando, porque a medicação está atrasada, que quer a medicação agora, urgente, porque o paciente está ruim, então até você tem que arrumar tudo correndo, tudo no hospital é urgente, é emergência, o paciente está morrendo. Então a farmácia, os funcionários, trabalham sobre pressão, é muita coisa, ele atende tudo, todas as clínicas, é um serviço apertado e corrido. Então é a falta de comunicação que está levando muitos erros para o paciente. (1TFC)
Problemas de administração e organização dos serviços, bem como, problemas relativos à estrutura física, financeira, ao sistema de medicação, desorganização de pessoas e unidades, problemas na gestão do pessoal e na administração e organização dos serviços da unidade, também foram identificados.

\section{Não sabemos a causa da troca de leitos dos pacientes pela enfermagem. (5TFC)}

Problemas estruturais. A estrutura física do hospital também propicia erros, não há lugar adequado para prescrever, na maioria dos locais são lugares barulhentos, apertados. (2MB)

Desorganização e descaso da diretoria e supervisões cooperam para que a farmácia funcione de maneira inadequada. (1AFB)

Falta de pessoal suficiente para cada atividade. (1FD)

Má capacitação dos profissionais. (1MRD)

Estudo $^{(16)}$ identificou os seguintes fatores organizacionais como causas dos erros: treinamento inadequado, baixa percepção da importância da prescrição, a hierarquia na equipe médica e a ausência de autoconsciência dos erros.

Ao responder sobre as providências clínicas ou administrativas tomadas nos hospitais, os dados organizados, estão apresentados na Tabela 3.

A Tabela 3 mostra o expressivo número de citações da realização de relatórios. O relatório dos erros, entretanto, não tem sido observado como um documento de análise de qualidade para a prevenção de futuros erros, mas como documento de punição do membro da equipe envolvido. Geralmente, os relatórios são de erros da equipe de enfermagem, sendo rara a realização destes para equipe médica e de farmácia. Ainda não há, em nenhuma dessas instituições, um comitê formal de avaliação de erros na medicação, o que limita qualquer documentação.

Quase sempre, os erros são punidos com advertência ou são encaminhados a comissões julgadoras que encaminham as ocorrências. O sistema não muda. (6EB)

E se os erros persistirem, advertência para o funcionário, para que não venha prejudicar o setor. Se não aprender, tem que chegar até a demissão. (1TFC)

Constata-se que as providências administrativas frente ao erro na medicação voltaram-se para o profissional que errou e não para o desenvolvimento de estratégias que transformem o erro em aprendizado para o sistema.

Estudo $^{(18)}$ aponta que, na ocorrência de um erro na medicação, não é dada ênfase à educação e sim, à punição e que isso, ao invés de ajudar a prevenir faz com que cada vez menos os erros sejam notificados, prejudicando o conheci- 
mento de seus fatores de risco e permitindo sua repetição. Há algumas citações em que, ao contrário, nenhuma providência é tomada.
Nunca observou nada a ser feito. Uma vez houve um residente que se irritou com a falta de remédios e foi conversar com a diretoria, mas nada adiantou. (1EB)

Não verifiquei a tomada de nenhuma providência"efetiva" na ocorrência desses erros. (7EB)

Tabela 3 - Distribuição das categorias de providências clínicas e/ou administrativas que são tomadas nos quatro hospitais, segundo a opinião dos entrevistados (Goiânia, Recife, Ribeirão Preto, São Paulo) - 2002

\section{CATEGORIA}

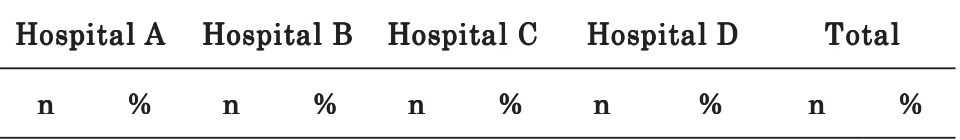

Providências Clínicas

Em relação ao paciente

Em relação aos profissionais

\begin{tabular}{|c|c|c|c|c|c|c|c|c|c|}
\hline- & - & 4 & 12,9 & 5 & 8,9 & 5 & 7,0 & 14 & 7,6 \\
\hline- & - & 10 & 32,3 & - & - & 1 & 1,4 & 11 & 6,0 \\
\hline 7 & 28 & - & - & 4 & 7,1 & - & - & 11 & 6,0 \\
\hline 5 & 20 & - & - & 14 & 25 & - & - & 19 & 10,4 \\
\hline 6 & 24 & - & - & 4 & 7,1 & - & - & 10 & 5,5 \\
\hline 3 & 12 & - & - & 4 & 7,1 & - & - & 7 & 3,8 \\
\hline 1 & 4,0 & - & - & - & - & - & - & 1 & 0,5 \\
\hline 1 & 4,0 & - & - & - & - & - & - & 1 & 0,5 \\
\hline- & - & - & - & 1 & 1,8 & - & - & 1 & 0,5 \\
\hline- & - & - & - & 9 & 16,1 & - & - & 9 & 4,9 \\
\hline 2 & 8 & 3 & 9,7 & 10 & 17,9 & - & - & 15 & 8,2 \\
\hline- & - & 3 & 9,7 & 1 & 1,8 & 1 & 1,4 & 5 & 2,7 \\
\hline- & - & 3 & 9,7 & - & - & 6 & 8,4 & 9 & 4,9 \\
\hline- & - & - & - & - & - & 17 & 23,9 & 17 & 9,3 \\
\hline- & - & - & - & 2 & 3,6 & 34 & 47,9 & 36 & 19,7 \\
\hline - & - & 8 & 25,8 & 1 & 1,8 & 7 & 9,9 & 16 & 8,7 \\
\hline - & - & - & - & 1 & 1,8 & - & - & 1 & 0,5 \\
\hline 25 & 100 & 31 & 100 & 56 & 100 & 71 & 100 & 183 & 100 \\
\hline
\end{tabular}

Providências Administrativas

Relatório

Orientação

Relatório e Orientação

Orientação e Advertência

Relatório, Orientação e Advertência

Relatório, Orientação e Suspensão

Relatório e Suspensão

Suspensão/Demissão

Advertência Verbal ou Escrita

Reuniões

Comunicados(Memorandos, notificações)

Comunicados em geral

Contatos e decisões pessoais

Nenhuma providência

Outros

Total

As informações, obtidas dos entrevistados, sobre as

Esse fato é uma tônica nos hospitais brasileiros não havendo estatísticas relatadas em publicações sobre a ocorrência dos erros na medicação devido à subnotificação destes erros pela equipe de saúde. sugestões para prevenir erros ou potencial para errar, são apresentadas na Tabela a seguir:

Tabela 4 - Distribuição das categorias de sugestões para evitar erros ou potenciais para errar em quatro hospitais investigados, segundo a opinião dos entrevistados (Goiânia, Recife, Ribeirão Preto, São Paulo) - 2002

\begin{tabular}{|c|c|c|c|c|c|c|c|c|c|c|}
\hline \multirow{2}{*}{ CATEGORIA } & \multicolumn{2}{|c|}{ Hospital A } & \multicolumn{2}{|c|}{ Hospital B } & \multicolumn{2}{|c|}{ Hospital C } & \multicolumn{2}{|c|}{ Hospital D } & \multicolumn{2}{|c|}{ Total } \\
\hline & $\mathrm{n}$ & $\%$ & n & $\%$ & $\mathbf{n}$ & $\%$ & n & $\%$ & $\mathbf{n}$ & $\%$ \\
\hline Educação Continuada & 6 & 17,1 & 4 & 4 & 14 & 15,2 & 19 & 19,8 & 43 & 15,2 \\
\hline Reestruturação no quadro de pessoal & 5 & 14,3 & 6 & 10,2 & 4 & 4,3 & 15 & 15,62 & 30 & 10,6 \\
\hline Melhores condições de trabalho e remuneração & - & - & 4 & 6,8 & 4 & 4,3 & 3 & 3,1 & 11 & 3,9 \\
\hline Informatização do Sistema & - & - & 11 & 18,6 & 7 & 7,6 & 11 & 11,5 & 29 & 10,3 \\
\hline Atitudes individuais & 8 & 22,9 & 1 & 1,7 & 26 & 28,3 & 15 & 15,6 & 50 & 17,7 \\
\hline Melhorar comunicação e interação entre & & & & & & & & & & \\
\hline setores e pessoas & 4 & 11,4 & 7 & 11,9 & 5 & 5,4 & 7 & 7,3 & 23 & 8,2 \\
\hline Aumentar supervisão, vigilância e punições & 1 & 2,9 & 1 & 1,7 & 10 & 10,9 & 6 & 6,2 & 18 & 6,4 \\
\hline Realização de reuniões & - & - & - & - & 3 & 3,3 & 4 & 4,2 & 7 & 2,5 \\
\hline Reestruturação do sistema e & & & & & & & & & & \\
\hline reorganização da clínica & 5 & 14,28 & 0 & 0 & 2 & 2,17 & 0 & 0 & 7 & 2,48 \\
\hline Outros & 1 & 2,86 & 14 & 23,73 & 10 & 10,87 & 8 & 8,33 & 33 & 11,70 \\
\hline Total & 35 & 100 & 59 & 100 & 92 & 100 & 96 & 100 & 282 & 100 \\
\hline
\end{tabular}


As sugestões dos profissionais para a prevenção dos erros na medicação estiveram voltadas para as alterações nas atitudes individuais como estratégias mais eficazes para prevenir erros. Ao analisar os demais resultados, percebemos que mais uma vez o indivíduo foi responsabilizado pelo sucesso ou insucesso da terapia medicamentosa. Os entrevistados indicam que as atitudes individuais como se atentar mais, melhorar a comunicação entre setores e pessoas e manter a letra legível favorecerá o trabalho de outras equipes como o da enfermagem e farmácia. Não podia ser diferente já que atribuem os erros à falta de atenção.

$\mathrm{Na}$ prescrição, você tem que pensar fazer a letra mais legível possível, tentar não deixar nada confuso ou em dúvida pra quem vai ler. (3MC)

A Educação Continuada desponta como uma das sugestões citadas.

Eu acho que a melhor forma de estar evitando erros seria estar sempre dando curso sobre administração, mais estudo, palestras. (2TEC)

Eu acho que deveria fazer um trabalho especial com essas pessoas, não aquelas palestras cansativas que as pessoas quase não vão, não prestam atenção. Deveria sim trazer pessoas pra passarem pelos procedimentos, de preparar a medicação, a pessoa acompanhar realmente funcionário na administração. Às vezes chega o funcionário com anos e anos de experiência, mas às vezes ele está tão acostumado tão cheio de vício de fazer errado que às vezes ele faz e nem percebe. (6TEC)

Obviamente, a contínua educação de todos os profissionais da equipe médica, de farmácia e enfermagem pode auxiliar nas atividades de segurança do paciente, mas ela deve estar envolvida com outras atividades relativas ao sistema. Nessa perspectiva, mudanças na estrutura hospitalar, nas condições de trabalho, na comunicação e interação entre setores e pessoas, a informatização do sistema, com adequação do sistema de prescrição eletrônica, implantação de dose unitária, são estratégias que realmente favoreceram a segurança dos pacientes e foram sugeridas nos relatos de alguns profissionais:

Serem prescritos medicamentos apenas eletronicamente. (3AAA)

Aumentar o quadro de funcionários para melhor atender o cliente. (6AEB)

\section{REFERÊNCIAS}

(1) Carvalho VT. Erros na administração de medicamentos: análise de relatos dos profissionais de enfermagem [dissertação]. Ribeirão Preto: Escola de Enfermagem de Ribeirão Preto/USP; 2000.
Implantação da dose unitária por horário e implantação da leitura por código de barras. (3FC)

Centralizando o médico pode ter um centro para buscar informação, poderá haver superação de vícios de prescrição e os médicos especificarão melhor a concentração e volume. (1FC)

Mudança no sistema de liberação de psicotrópicos. (1MB)

Melhora no espaço físico. (1AFB)

Reuniões multidisciplinares entre enfermagem, médicos e farmácia. (9MC)

\section{CONCLUSÕES}

Este estudo identificou e analisou os tipos, causas, providências administrativas e sugestões a respeito de erros na medicação, na perspectiva dos profissionais envolvidos no sistema de medicação, em quatro hospitais de diferentes regiões brasileiras.

Constatou-se que os erros relacionados à prescrição/ transcrição dos medicamentos foram os mais citados pelos profissionais. A falta de atenção, falhas individuais e problemas na administração dos serviços constituíram importantes atributos das causas dos erros.

Na presença dos erros, são tomadas providências clínicas e administrativas (relatórios e orientação ao membro da equipe envolvido). Entretanto, esses relatórios não são utilizados para uma avaliação de qualidade ou para melhorias no sistema, na maior parte das vezes vão acabar no prontuário do funcionário. Os quase-erros ou erros em potencial, aqueles erros interceptados antes do paciente ser atingido, não são nem considerados erros, quanto mais notificados. Perpetuam-se, assim, as condições falhas do sistema.

Dentro da cultura organizacional que identifica erros como causas individuais, a sugestão dos entrevistados é que mudanças nas atitudes individuais e educação continuada poderiam solucionar o problema.

Enfim, este estudo evidenciou a gama de pontos vulneráveis do processo de administração de medicamentos nos hospitais investigados. Sugere-se, assim, uma reflexão urgente sobre os aspectos abordados para que medidas proativas sejam implementadas. A melhoria da qualidade é imperativa nesse sentido visando a segurança dos pacientes.

(2) Barker KN, Flyin EA, Pepper GA, Bates DW, Mikeal RL. Medication errors observed in 36 health care facilities. Arch Intern Med. 2002;162(16):1897-903. 
(3) Berwick DM, Leape LL. Reducing errors in medicine. BMJ. 1999;319(3):136-7.

(4) Stefl ME. To err is human: building a safer health system in 1999. Front Health Serv Manage. 2001;18(1):1-2.

(5) Anderson JG, Jay SJ, Anderson M, Hunt TJ. Evaluating the capability of information technology to prevent adverse drug events: a computer simulation approach. J Am Med Inform Assoc. 2002;9(5):479-90.

(6) Bueno E, Cassiani SHB, Miquelim JL. Erros na administração de medicamentos: fatores de risco e medidas empregadas. Rev Baiana Enferm. 1998;11(1):101-19.

(7) Cassiani SHB. Erros na administração de medicamentos: estratégias de prevenção. Rev Bras Enferm. 2000;53(3):424-30.

(8) Anderson DJ, Webster CA. Systems approach to reduction of medication error on the hospital ward. J Adv Nurs. 2001;35(1):34-41.

(9) Bates DW, Cohen M, Leape LL, Overhage JM, Shabot MM, Sheridan T. Reducing the frequency of errors in medicine using information technology. J Am Med Inform Assoc. 2001; 8(4):299-308.

(10) Cassiani SHB, Freire CC, Gimenes FRE. A prescrição médica eletrônica em um hospital universitário: falhas de redação e opiniões de usuários. Rev Esc Enferm USP. 2003;37(4):51-60.
(11) Bates DW. Improving medication safety across institutions. J Qual Improvem. 2000;26(6): 319-20.

(12) Jyh JH. Avaliação do conhecimento farmacoterápico de médicos e graduandos em medicina humana [tese]. Botucatu: $\mathrm{Fa}-$ culdade de Medicina de Botucatu da UNESP; 2003.

(13) Moscati IM, Persano S, Castro LLC. Aspectos metodológicos e comportamentais da adesão à terapêutica. In: Castro LLC. Fundamentos de farmacoepidemiologia. São Paulo: AG; 2000. p. 171-80.

(14) Leape LL, Bates DW, Cullen DJ, Cooper J, Demonaco HJ, Gallivan T, et al. System analysis of adverse drug events. JAMA. 1995;274(1):35-43.

(15) Cohen MR, editor. Medication errors: causes, prevention, and risk management. Boston: Jones and Bartlett; 2000.

(16) Dean B, Schachter M, Vincent C, Barber N. Causes of prescribing errors in hospital inpatients: a prospective study. Lancet. 2002;359(9315):1373-8.

(17) LaPointe NMA, Jollis JG. Medication errors in hospitalized cardiovascular patients. Arch Intern Med. 2003;163(12): 1461-6.

(18) Cohen MR. Medication-error reporting: banish a system that blames. Nursing. 1996;26(1):15. 